\title{
(2) OPEN ACCESS \\ Treatments for dry age-related macular degeneration: therapeutic avenues, clinical trials and future directions
}

\author{
Thales Antonio Cabral de Guimaraes (D) ,',2 Malena Daich Varela (D) , 1,2 \\ Michalis Georgiou (D) ,', Michel Michaelides ${ }^{1,2}$
}

'Institute of Ophthalmology, University College London, London, UK

${ }^{2}$ Moorfields Eye Hospital NHS Foundation Trust, London, UK

\section{Correspondence to}

Michel Michaelides, Moorfields Eye Hospital NHS Foundation Trust, London EC1V 2PD, UK; michel.michaelides@ucl.ac.uk

Received 19 November 2020 Revised 20 January 2021 Accepted 23 February 2021

Check for updates

(c) Author(s) (or their employer(s)) 2021. Re-use permitted under CC BY. Published by BMJ.

\section{To cite: Cabral de} Guimaraes TA, Daich

Varela M, Georgiou M, et al. Br J Ophthalmol Epub ahead of print: [please include Day Month Year]. doi:10.1136/ bjophthalmol-2020-318452

\begin{abstract}
Age-related macular degeneration (AMD) is the leading cause of irreversible blindness in the developed world. The identification of the central role of vascular endothelial growth factor (VEGF) in the pathogenesis of neovascular AMD and the introduction of anti-VEGF agents as gold-standard treatment, have drastically changed its prognosis - something yet to be seen in dry AMD. Several therapeutic avenues with a wide variability of targets are currently being investigated in dry AMD. The approaches being investigated to reduce the rate of disease progression include, (1) drugs with antioxidative properties, (2) inhibitors of the complement cascade, (3) neuroprotective agents, (4) visual cycle inhibitors, (5) gene therapy and (6) cell-based therapies. A number of early phase clinical trials have provided promising results, with many more ongoing and anticipated in the near future. In this review, we aim to provide an update of the interventional trials to date and future prospects for the treatment of dry AMD.
\end{abstract}

\section{INTRODUCTION}

Age-related macular degeneration (AMD) is a multifactorial condition and the leading cause of irreversible blindness in the elderly population, with the number of affected patients predicted to be 288 million by $2040 .{ }^{12}$ Its progressive and irreversible nature translates to a significant societal cost burden and increased health resource utilisation. ${ }^{3-5}$ Moreover, ageing is the main risk factor to develop AMD, hence, as life expectancy increases, this burden will ultimately become greater in the foreseeable future.

There are several classifications of AMD, based on clinical and imaging findings. ${ }^{6-8}$ One published approach suggests that early and intermediate stages are characterised by variable size and amount of drusen and the presence of pigmentary abnormalities, and the late stage displays signs of advanced disease, such as geographic atrophy (GA) and neovascularisation. ${ }^{9}$ AMD can be pragmatically divided into two groups according to the presence or absence of neovascularisation: (1) dry or nonneovascular AMD (or GA) and (2) wet or neovascular AMD (nAMD). The implication of vascular endothelial growth factor (VEGF) in the pathogenesis of nAMD and the introduction of anti-VEGF as the gold-standard treatment has drastically changed its prognosis-something yet to be seen in dry AMD.
Several therapeutic approaches have been investigated for dry AMD and are currently in different stages of clinical trial, including antioxidative therapy, drug treatments targeting multiple pathways, cell and gene directed therapies, as well as retinal implants. This article aims to provide an update on prioritised current therapeutic avenues for dry AMD and future treatment prospects.

\section{PATHOGENESIS OF DRY AMD}

AMD is a complex multifactorial disease whose pathogenesis is incompletely understood and evolving, with a detailed evaluation behind the scope of this review. Several factors are believed to contribute to its pathogenesis, including genetic, ${ }^{10}$ oxidative stress, ${ }^{11}$ environmental, ${ }^{12-14}$ inflammatory ${ }^{15}$ and ischaemic. ${ }^{16}$ The presence of drusen is considered the hallmark of earlier stages of AMD, which may enlarge, become confluent and evolve to drusenoid retinal pigmented epithelium (RPE) detachments. ${ }^{17}$ This causes a disruption in the interaction between Bruch's membrane and RPE, inhibiting RPE function, including the crucial ability to transport photoreceptor debris across Bruch's membrane to the choriocapillaris, further facilitaing accumulation of lipofuscin and other related products including A2E. ${ }^{18}$

Drusen contain several proinflammatory factors including complement pathway components (and lipofuscin-related products), which have been identified as one of the main contributors to AMD development. ${ }^{19}$ Several studies suggest that complement pathway activation and the consequent membrane attack complex (MAC) play a key role in choriocapillaris loss and in the development of AMD and GA. ${ }^{20-22}$ Mullins et al correlated vascular loss and choriocapillaris density with the extent of drusen, suggesting that in early stages, the deposition of complement complexes induces loss of choriocapillaris and drusen formation. ${ }^{23}$ Subsequently, the same group observed the deposition of MAC in the outer part of Bruch's membrane prior to choriocapillaris loss and identified that the high-risk allele $\mathrm{Y} 402 \mathrm{H}$ in $\mathrm{CFH}$ (OMIM \#134370) is associated with elevated levels of MAC and increased risk of choriocapillaris loss in early AMD disease. ${ }^{24}$ Polymorphisms in multiple other genes have also been associated with increased risk of developing $\mathrm{AMD}$, including genes involved in the complement cascade, ARMS2 (OMIM \#611313), ABCA4 (OMIM \#601691), and HTRA1 (OMIM 
Table 1 Summary of ongoing clinical trials targeting dry AMD

\begin{tabular}{|c|c|c|c|c|c|c|c|}
\hline Drug category/name & $\begin{array}{l}\text { Clinical trial ID } \\
\text { (NCT \#) }\end{array}$ & Study phase & Route of delivery & Status & Sponsor & Location & $\begin{array}{l}\text { Number of } \\
\text { patients }\end{array}$ \\
\hline \multicolumn{8}{|l|}{ Antioxidative } \\
\hline AREDS & NCT00000145 & Phase III & Oral & Completed & National Eye Institute (NEI) & USA & 3640 \\
\hline AREDS2 & NCT00345176 & Phase III & Oral & Completed & National Eye Institute (NEI) & USA & 4203 \\
\hline OT-551 & NCT00306488 & Phase II & Topical & Completed & $\begin{array}{l}\text { National Institutes of Health } \\
\text { Clinical Center (CC) }\end{array}$ & USA & 11 \\
\hline \multicolumn{8}{|c|}{ Reduction of toxic bryproducts } \\
\hline GSK933776 & NCT01342926 & Phase II & IV & Completed & GlaxoSmithKline & USA & 191 \\
\hline RN6G & NCT01577381 & Phase II & IV & Terminated & Pfizer & USA & 10 \\
\hline \multicolumn{8}{|l|}{ Visual cycle modulators } \\
\hline ACU-4429 & NCT01802866 & Phase Ilb/III & Oral & Completed & Kutoba Vision Inc & USA & 508 \\
\hline Fenretinide & NCT00429936 & Phase II & Oral & Completed & Sirion Therapeutics, Inc & USA & 246 \\
\hline C20-D3-vitamin A (ALK-001) & NCT03845582 & Phase III & Oral & Ongoing—recruiting & Alkeus Pharmaceuticals, Inc & USA & 300 \\
\hline \multicolumn{8}{|c|}{ Anti-inflammatory and complement inhibition } \\
\hline Eculizumab & NCT00935883 & Phase II & IV & Completed & Philip J. Rosenfeld, MD & USA & 30 \\
\hline Lampalizumab & NCT02247531 & Phase III & Intravitreal & Terminated & Hoffman-La Roche & Multicenter & 906 \\
\hline Lampalizumab & NCT02247479 & Phase III & Intravitreal & Terminated & Hoffman-La Roche & Multicenter & 975 \\
\hline Sirolimus (rapamycin) & NCT00766649 & Phase I/II & Subconjunctival & Completed & National Eye Institute (NEI) & USA & 11 \\
\hline Avacincaptad pegol (Zimura) & NCT02686658 & Phase II/III & Intravitreal & Completed & IVERIC bio, Inc. & Multicenter & 286 \\
\hline Pegcetacoplan (APL-2) & NCT02503332 & Phase II & Intravitreal & Completed & Apellis Pharmaceuticals Inc. & Multicenter & 246 \\
\hline Pegcetacoplan (APL-2) & NCT03525600 & Phase III & Intravitreal & Ongoing-not recruiting & Apellis Pharmaceuticals Inc. & Multicenter & 600 \\
\hline Pegcetacoplan (APL-2) & NCT03525613 & Phase III & Intravitreal & Ongoing-not recruiting & Apellis Pharmaceuticals Inc. & Multicenter & 600 \\
\hline Tedisolumab (LFG316) & NCT01527500 & Phase II & Intravitreal & Completed & Novartis Pharmaceuticals & USA & 150 \\
\hline Risuteganib & NCT03626636 & Phase II & Intravitreal & Completed & Allegro Ophthalmics & USA & 42 \\
\hline \multicolumn{8}{|l|}{ Neuroprotection } \\
\hline Ciliary nerve trophic factor & NCT00063765 & Phase I & Intravitreal & Completed & National Eye Institute (NEI) & USA & 10 \\
\hline Ciliary nerve trophic factor & NCT00447954 & Phase II & Intravitreal & Completed & Neurotech Pharmaceuticals & USA & 51 \\
\hline Brimonidine tartrate & NCT00658619 & Phase II & Intravitreal & Completed & Allergan & Multicenter & 113 \\
\hline Brimonidine tartrate & NCT02087085 & Phase Ilb & Intravitreal & Terminated & Allergan & Multicenter & 303 \\
\hline \multicolumn{8}{|l|}{ Gene therapy } \\
\hline AAVCAGsCD59 & NCT03144999 & Phase I & Intravitreal & Completed & Hemera Biosciences & USA & 17 \\
\hline GT005 & NCT03846193 & Phase I/II & Subretinal & Ongoing - Recruiting & Gyroscope Therapeutics & UK & 35 \\
\hline \multicolumn{8}{|l|}{ Cell-based therapies } \\
\hline Palucorcel (CNTO-2476) & NCT01226628 & Phase I/II & Subretinal & Completed & $\begin{array}{l}\text { Janssen Research \& Development, } \\
\text { LLC }\end{array}$ & USA & 35 \\
\hline MA09-hRPE & NCT01344993 & Phase I/II & Subretinal & Completed & $\begin{array}{l}\text { Astelas Institute for Regenerative } \\
\text { Medicine }\end{array}$ & USA & 9 \\
\hline CPCB-RPE1 & NCT02590692 & Phase I/lla & Subretinal & Ongoing—not recruiting & Regenerative Patch Technologies & USA & 16 \\
\hline \multicolumn{8}{|l|}{ Mitochondrial enhancers } \\
\hline Elamipretide & NCT02848313 & Phase I & Subcutaneous & Completed & Stealth Biotechnologies Inc & USA & 40 \\
\hline Elamipretide & NCT03891875 & Phase II & Subcutaneous & Ongoing—recruiting & Stealth Biotechnologies Inc & USA & 180 \\
\hline \multicolumn{8}{|l|}{ Nanosecond laser therapy } \\
\hline 2RT nanosecond laser & NCT01790802 & $\begin{array}{l}\text { Not } \\
\text { applicable }\end{array}$ & $\begin{array}{l}\text { Retinal active laser } \\
\text { therapy }\end{array}$ & Completed & Center for Eye Research Australia & Australia & 292 \\
\hline
\end{tabular}

AMD, age-related macular degeneration.

\#602194). ${ }^{2526}$ It is still unclear if these genetic risk alleles are associated with the development of either wet or dry AMD, or both.

\section{TREATMENTS FOR DRY AMD}

Currently, the management of dry AMD consists of observation, regular follow-up evaluations and documentation, for timely recognition of visual function deterioration with appropriate rehabilitation and early choroidal neovascularisation (CNV) detection. Several therapeutic avenues are being investigated aiming at: (1) disease prevention, (2) halting disease progression and/or (3) restoration of vision. In this section, we summarise the most promising pathways of treatment and the ongoing clinical trials (table 1 ).

\section{Nutritional supplementation and antioxidant therapy}

Oxidative damage from various sources, such as smoking, UV light exposure and oxidative stress to the retina have been strongly linked with AMD. Hence, treatments that reduce the accumulation of reactive oxygen species may be a potential therapeutic intervention.

Age-related eye disease study (AREDS) supplements

AREDS was a double-masked, randomised and multicentre trial $(n=3640$; NCT00000145) that was designed to determine the protective effects of antioxidant supplementation in patients with AMD. The participants were divided into four groups based on disease severity. The results showed that a daily dose of 
vitamin E (400 IU), C (500 mg), Beta-carotene (15 mg), cupric acid $(2 \mathrm{mg})$ and zinc oxide $(80 \mathrm{mg})$ reduced the odds of developing advanced AMD in up to $34 \%$ of the subjects with high-risk characteristics (groups 3 and 4). Moreover, follow-up of these participants over a 12-year period demonstrated that subjects who had the highest omega-3 fatty acids intake were 30\% less likely to develop central GA and nAMD. ${ }^{27}$

Subsequently, in 2006, a new placebo-controlled phase III AREDS study (AREDS2; $\mathrm{n}=4203$; NCT00345176) commenced to determine whether adding lutein + zeaxanthin, omega-3 longchain polyunsaturated fatty acids (docohexaenoic acid (DHA) and eicosapentaenoic acid (EPA)), or a combination of both to the AREDS formulation would decrease the risk of developing advanced AMD. Addition of lutein + zeaxanthin, DHA + EPA, or both, to the AREDS formulation in primary analyses did not further reduce risk of progression to advanced AMD. More lung cancers, mostly in former smokers, were noted in the betacarotene group $(n=23)$, when compared with the group without $(n=11)$. This finding led to the substitution of the carotenoid for lutein + zeaxanthin in the AREDS formula. ${ }^{28} 29$ In 2017, a pooled systematic meta-analysis concluded that, although some patients with higher risk of progression to more advanced stages of AMD may experience delay in progression with antioxidant vitamin and mineral supplementation, lutein and zeaxanthincontaining supplements may have little to no effect on the disease progression. ${ }^{30}$

\section{1-hydroxy-4-cyclopropanecarbonyloxy-2,2,6,6-tetramethylpiperidine} hydrochloride (OT-551)

OT-551 is a molecule with anti-inflammatory and antioxidant effects that was found to protect against light-induced degeneration in the RPE of rats. ${ }^{31}$ An open-label phase II trial $(n=11$; NCT00306488) administered topical $0.45 \%$ OT-551 in a randomly assigned eye of participants with bilateral GA. No serious adverse events were noted in the 11 participants. The mean change in best-corrected visual acuity (BCVA) at 2 years was $+0.2 \pm 13.3$ letters in the study eye versus $-11.3 \pm 7.6$ letters in the non-study eye. No statistical significant differences were found between the study and contralateral eye in microperimetry measurements, contrast sensitivity, area of GA and total drusen area from baseline, with benefits deemed as limited or without benefit, in the delivered mode and concentration. ${ }^{32}$

\section{Reduction of toxic byproducts $\beta$-amyloid}

Another pathway being explored is targeting $\beta$-amyloid, a major endogenous protein underlying the aetiology of, for example, Alzheimer's disease. ${ }^{33}$ It has been identified as a component of drusen and it is a known activator of the complement cascade. In a placebo-controlled phase II study $(n=191$; NCT01342926) conducted in patients with GA secondary to AMD, intravenous transfusion of GSK933776 (an anti-amyloid $\beta$ monoclonal antibody) was deemed well-tolerated and safe. However, there was no clinically meaningful improvement in visual function or decrease in the rate of GA enlargement. ${ }^{34}$ Similarly, RN6G, another antiamyloid $\beta$ monoclonal antibody has been evaluated in a phase II placebo-controlled trial $(n=10$; NCT01577381), administered intravenous in patients with GA. The study was stopped early by the sponsor, and not enough subjects/data were recruited/collected for meaningful analyses. ${ }^{35}$

GAL-101 (formerly known as MRZ-99030) is a dipeptide that has been shown to prevent the formation of oligomeric $\beta$-amyloid species. ${ }^{36}$ In a phase I trial $(n=70$; NCT01714960),
GAL-101 was administered as an eyedrop in subjects with glaucoma. The drug was deemed safe, with a low frequency of side effects. ${ }^{37}$ A phase II trial is in development to determine its safety and efficacy in both glaucoma and dry AMD. ${ }^{38}$

\section{Visual cycle modulators}

Modulation of the visual cycle by targeting participating enzymes, delivery of vitamin A to the cycle or removal of associated toxic waste products may be beneficial.

\section{Emixustat hydrochloride}

Oral administration of ACU-4429 (emixustat hydrochloride) has been investigated in the SEATTLE study $(n=508$; NCT01802866), a multicentre, randomised, phase IIb/III trial. The primary efficacy endpoint was the mean annual growth rate of total GA area, as measured by fundus autofluorescence (FAF). Although the drug was found to be safe, on average, GA lesions in the treated group progressed at a similar rate to the placebo group. ${ }^{39}$

\section{Fenretinide}

Fenretinide is a synthetic derivative of vitamin A that binds to the serum retinol-binding protein (RBP), allowing a rapid elimination of fenretinide-RBP complex through urine. In animal models, it was found to reduce circulating RBP-retinol complex levels in a dose-dependent manner and lower the production of $\mathrm{A} 2 \mathrm{E}$ in abca $4^{-1-}$ mice. ${ }^{40} \mathrm{~A}$ phase II, placebo-controlled, proof of concept trial, studied the efficacy of oral fenretinide in patients with GA due to AMD ( $n=246$; NCT00429936). The drug was found to be safe but associated with side effects including significantly delayed dark adaptation. However, the achieved reduction of GA growth rate in treated patients (both high and low dose) was not statistically significant, compared with the placebo group. ${ }^{41}$

\section{Deuterated vitamin A}

A modified form of vitamin $\mathrm{A}$, with a deuterium isotope replacement at carbon 20 (C20-D3-vitamin A), was designed to reduce the rate at which retinaldehyde reacts, reducing dimerisation and thereby potentially decreasing the accumulation of toxic byproducts. ${ }^{42}$ Administration of C20-D3-vitamin A in rodents with no genetic defects in vitamin A processing, decreased the rate of A2E biosynthesis. ${ }^{43}$ A randomised, placebo-controlled phase III study (SAGA study) of oral ALK-001(C20-D3-vitamin A) in subjects with GA due to AMD ( $n=300$; NCT03845582) has commenced; with an estimated primary completion date of December 2021.

\section{Anti-inflammatory agents and complement inhibitors}

Several treatment strategies that modulate the complement system in patients with AMD are currently being investigated (figure 1).

\section{Eculizumab}

Eculizumab is an IgG antibody involved in the inhibition of complement component 5 (C5) that has been FDA-approved for the treatment of atypical haemolytic uremic syndrome and paroxysmal nocturnal haemoglobinuria, both resulting from complement cascade dysregulation. ${ }^{44} 45$ The COMPLETE study $(\mathrm{n}=30$; NCT00935883) was a prospective, double-masked, randomised phase II clinical trial that evaluated the safety and efficacy of intravenous eculizumab for reducing the growth of GA in AMD. ${ }^{46}$ No drug-related adverse events were identified 


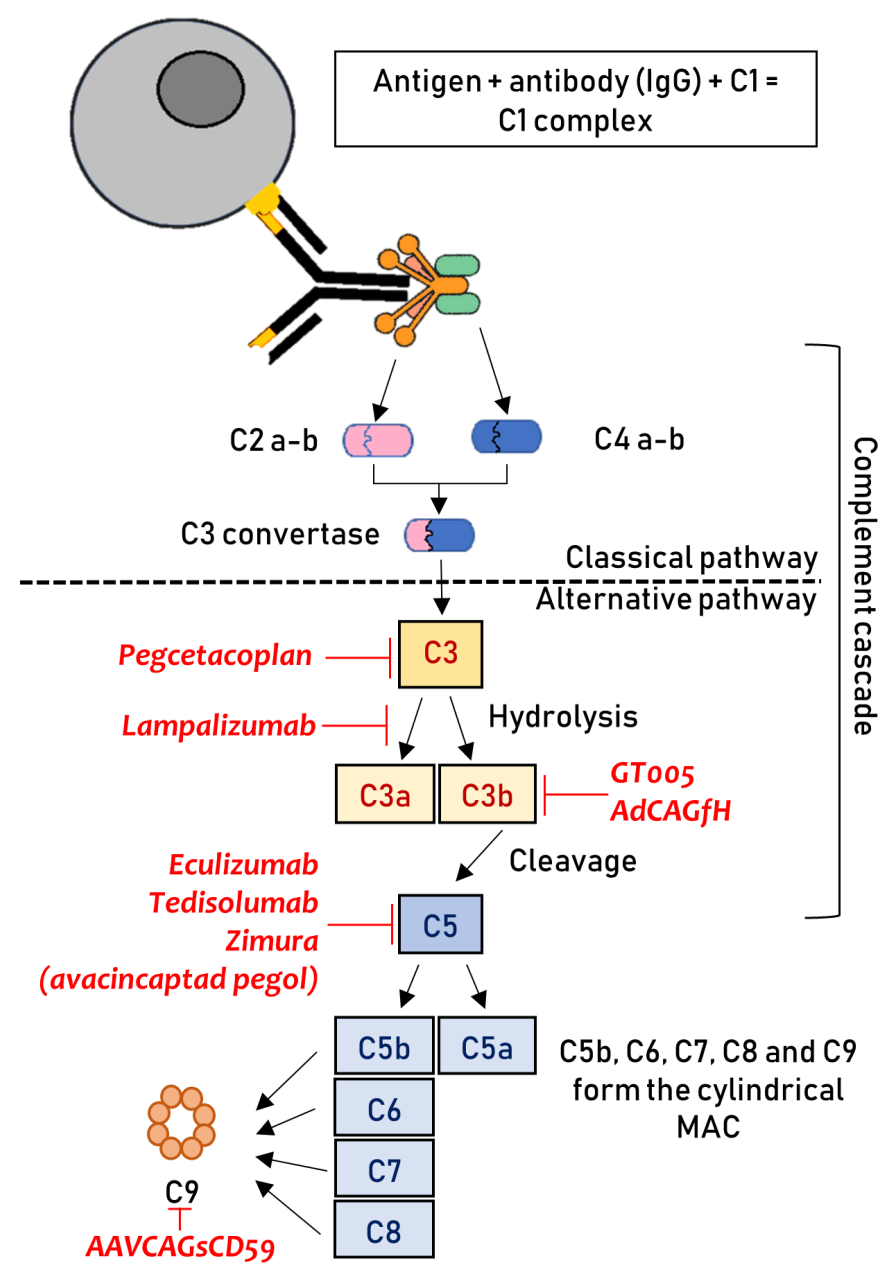

Figure 1 Graphical representation of the complement cascade. The location where different therapeutics act is highlighted. The therapies are shown, with them all have a downregulating effect on the particular step, negatively impacting on the downstream pathway. This figure has been created by the authors. C, complement; IgG, immunoglobulin G; MAC, membrane attack complex.

and none of the eyes converted to nAMD during a follow-up of 52 weeks. The drug did not decrease the growth rate of GA significantly; GA enlarged by a mean of $0.37 \pm 0.22 \mathrm{~mm}$ in the eculizumab-treated eyes and by a mean of $0.37 \pm 0.21 \mathrm{~mm}$ in the placebo group $\left(p=0.93,2\right.$ sample t-test). ${ }^{46}$

\section{Lampalizumab}

Lampalizumab is an antigen-binding fragment of a humanised monoclonal antibody that inhibits complement factor D. ${ }^{47} 48$ This factor has a key role in the alternative complement pathway, enabling the formation of $\mathrm{C} 3 \mathrm{~b}$ or C5 convertase. The CHROMA ( $n=906$; NCT02247479) and SPECTRI $(n=975$; NCT02247531) trials were two identically designed, phase III, randomised controlled trials that administered $10 \mathrm{mg}$ of intravitreal lampalizumab and the largest GA trials conducted to date. Although it was deemed safe, it did not reduce GA enlargement as compared with the sham group during the 48 weeks of follow-up. ${ }^{49}$

\section{Sirolimus}

Sirolimus (rapamycin) is a mammalian target of rapamycin inhibitor and immunosuppressive agent that is used for preventing organ rejection. ${ }^{50}$ It was administered subconjunctivally in a phase I/II trial ( $n=11$; NCT00766649) in eyes of patients with GA. Subjects received $440 \mu \mathrm{g}$ of subconjunctival sirolimus every 3 months. The investigational agent was well tolerated in all patients, but no positive anatomical or functional effects were identified and the rate of GA area growth did not decrease in the treated eyes as compared with the contralateral eyes. ${ }^{51}$

\section{Avacincaptad pegol}

Zimura (avacincaptad pegol) is an anti-C5 aptamer designed to decrease the activation of inflammasomes and the formation of MAC. A phase II/III, randomised controlled trial $(n=286$; NCT02686658; GATHER1) assessed the safety and efficacy of intravitreal administration of Zimura in subjects with GA. Published data showed that the investigational product met its prespecified primary endpoint-defined as reduction in the rate of GA growth measured by $\mathrm{FAF}$ - and was granted fasttrack designation by the FDA. ${ }^{52}$ Over a follow-up of 12 months, patients treated with a $2 \mathrm{mg}$ and $4 \mathrm{mg}$ dose achieved a $27.3 \%(\mathrm{p}$ value $=0.0072$ ) and $27.8 \%$ reduction ( $\mathrm{p}$ value $=0.0051$ ), respectively, in the mean rate of GA growth as compared with the sham group. ${ }^{53} \mathrm{CNV}$ was reported in the fellow eyes of 10 participants $(3.5 \%)$, in the study eye of 3 participants $(2.7 \%)$ in the sham cohort, 1 participant (4\%) in the avacincaptad pegol $1 \mathrm{mg}$ cohort, 6 participants (9\%) in the $2 \mathrm{mg}$ cohort and in 8 participants (9.6\%) in the $4 \mathrm{mg}$ cohort. Eighteen-month follow-up data are in keeping with aforementioned 12-month data, with a reduction in the mean rate of GA progression of $28.1 \%$ in the $2 \mathrm{mg}$ group and $29.9 \%$ in the $4 \mathrm{mg}$ group. ${ }^{54}$ Avacincaptad pegol was generally well tolerated and the adverse events were deemed to be related to the injection procedure. ${ }^{52}$ A second confirmatory phase III trial is underway (GATHER2).

\section{Pegcetacoplan}

Pegcetacoplan (APL-2) inhibits the complement component 3 (C3). FILLY was a phase II, multicentre, placebo-controlled, randomised trial $(n=246$; NCT02503332) that studied the safety and efficacy of intravitreal APL-2 in patients with GA. Patients receiving pegcetacoplan monthly or every other month (EOM), showed a GA growth rate reduction of $29 \%(\mathrm{p}=0.008)$ and $20 \%(p=0.067)$, respectively, as compared with the sham treatment group, ${ }^{55}$ although no differences were observed in BCVA outcomes between the groups. Adverse events included an increased incidence of exudation in treated eyes of $8.9 \%$ in the EOM group and $20.9 \%$ in the monthly group. Two patients in the monthly group and one subject in EOM group developed endophthalmitis. The FDA granted pegcetacoplan fast-track designation, and enrolment for the DERBY (NCT03525600) and OAKS (NCT0355613) phase III studies are now completed. ${ }^{56}$

\section{Tedisolumab}

A phase II, randomised trial $(n=150$; NCT01527500) administered intravitreal LFG316 (tesidolumab), a complement component 5 (C5) inhibitor, in patients with GA. After 12 months of follow-up, there was no statistically significant difference in GA area-the primary endpoint-between the treated and sham groups. There were no serious adverse events related to the study drug. ${ }^{57}$

\section{Risuteganib}

Risuteganib is an intravitreally administered integrin inhibitor, which binds and inhibits certain types of integrin heterodimers that are thought to be involved in the development of AMD. ${ }^{58}$ In a phase II, placebo-controlled study, $1 \mathrm{mg}$ of risuteganib was 
administered in patients with intermediate non-exudative AMD (NCT03626636; $n=42$ ). The primary endpoint was defined as the percentage of the population with $\geq 8$ letters ETDRS BCVA gain from baseline to week 28 versus from baseline to week 12 in the sham arm. The results showed that the primary endpoint was met in $48 \%$ of patients in the risuteganib group compared with $7.1 \%$ in the sham group, which was statistically significant. No serious adverse events were reported. ${ }^{59}$

\section{Neuroprotective agents}

Neuroprotection is another avenue with positive results in preclinical studies. If viable, it could lead to reduction of apoptosis and eventually halt the progression of GA in AMD. These drugs include antiapoptotic agents like tauroursodeoxycholic acid (TUDCA), ${ }^{60-62}$ dopamine-related therapies ${ }^{63}$ and growth factors such as ciliary neurotrophic factor (CNTF), ${ }^{64}$ among others. $^{65}$

\section{Ciliary neurotrophic factor (CNTF)}

Evidence from preclinical studies encouraged a phase I trial $(n=10 ;$ NCT00063765) to evaluate the safety of CNTF delivered over a 6-month period by encapsulated cells implanted intravitreally. The drug was deemed safe, with side effects being limited to low-grade anterior chamber activity that did not require treatment and a surgery-related choroidal detachment that resolved after the administration of topical steroids. ${ }^{66}$ Similarly, a placebocontrolled phase II study ( $\mathrm{n}=51$; NCT00447954), administered CNTF with the encapsulated cell technology intravitreally. The treatment group showed no difference in GA area or improvement in BCVA. ${ }^{67}$

\section{Brimonidine tartrate}

Brimonidine is an alpha2-adrenergic receptor agonist that has been established as an intraocular pressure-lowering agent, but promising evidence from clinical trials also suggests it has neuroprotective properties. ${ }^{68} 69$ It has been shown previously to promote survival and reduce photoreceptor damage in several animal models of acute retinal ischaemia, ${ }^{70} 71$ partial optic nerve crush $^{72}$ chronic ocular hypertension ${ }^{73}$ and in a light-induced model of progressive GA. ${ }^{74}$

The Brimonidine Drug Delivery System (Brimo DDS) is a sustained-release intravitreal implant. It was used in a randomised, placebo-controlled phase II trial $(\mathrm{n}=113$; NCT00658619), in which study eyes were treated either with $132 \mu \mathrm{g}, 264 \mu \mathrm{g}$ or sham procedure. GA area growth at month 12 was $1.78 \mathrm{~mm}^{2}$, $1.59 \mathrm{~mm}^{2}$ and $2.19 \mathrm{~mm}^{2}$ in each group, respectively, which was statistically significant $(p \leq 0.032)$. Adverse events were deemed as mild and related to the injection procedure. ${ }^{75}$ Subsequently, a larger placebo-controlled phase IIb study (BEACON study; $\mathrm{n}=303$; NCT02087085) used a new generation device with a higher dose $(400 \mu \mathrm{g})$ administered every 3 months in patients with GA. At 24 and 30 months of follow-up, GA area growth was reduced by $10 \%$ and $12 \%$, respectively, in the treatment group. The study was terminated early due to a slow GA progression rate of $\sim 1.6 \mathrm{~mm}^{2} /$ year in the enroled population. However, the drug significantly reduced GA growth at the 30 months analysis, with mild adverse events which were transient and limited to the injection site ${ }^{76}$; thereby resulting in an ongoing phase III trial. ${ }^{77}$

\section{Gene therapy}

Another treatment modality being explored is gene therapy. Due to the multifactorial and complex nature of AMD, there are multiple therapeutic challenges. Gene therapy interventions in AMD focus on the sustain expression of antiangiogenic and anticomplement proteins.

\section{Targeting MAC formation}

Two active phase I trials are using intravitreal AAVCAGsCD59 for both dry AMD and nAMD (NCT03144999 and NCT03585556, respectively). This compound acts as a membrane-bound inhibitor that reduces MAC formation (figure 1). ${ }^{78}$ It was previously shown to be successful in the attenuation of laser-induced CNV in mouse models, in both subretinal and intravitreal modes of delivery. ${ }^{79}$ Interim results of NCT03144999 (n=17) demonstrate the treatment to be well tolerated, with no dose-limiting toxicity. Four $(23.5 \%)$ eyes developed mild inflammation that resolved with topical steroids or observation, and two of these four subjects also required topical medication to treat raised intraocular pressure. ${ }^{80}$

A different MAC approach is being evaluated in an ongoing phase I/II trial to study the safety and efficacy of subretinal GT005 $(\mathrm{n}=35$; NCT03846193). GT005 was also designed to regulate complement activation and the formation of MAC (figure 1). It consists of a recombinant non-replicating adeno-associated viral (AAV) vector encoding complement factor I (a down-regulator of the C3b breakdown cycle), as a means to downregulate the alternative pathway. ${ }^{81}$ Subretinal administration of GT005 has been shown to significantly impact complement-driven $\mathrm{CNV}$ in a mouse laser-induced CNV model. ${ }^{82}$ The study is currently ongoing and has an estimated primary completion date of June 2021.

\section{Complement factor $\mathrm{H}$}

Subretinal AAV-mediated expression of complement factor $\mathrm{H}$ (AdCAGfH, OMIM \#134370) has been shown to attenuate C3-induced pathology in murine models, which is now under development for gene therapy in human subjects. ${ }^{83}$

\section{Cell-based therapies}

\section{Palucorcel}

Palucorcel (CNTO-2476) is a human umbilical cord tissuederived cell compound that has been previously shown to reduce functional deterioration in a rat model of retinal disease. ${ }^{84} \mathrm{~A}$ phase I/II dose-escalation study ( $\mathrm{n}=35$; NCT01226628), where the subretinal administration of CNTO-2476 via an ab externo surgical approach using the iTrack Model 275 microcatheter was evaluated in patients with GA. There was a high rate of adverse events related to the delivery procedure-17.1\% (6/35) of subjects experienced retinal detachments and 37.1\% (13/35) experienced retinal perforations-although, after 1 year, $34.5 \%$ $(10 / 29)$ and $24.1 \%(7 / 29)$ experienced $a \geq 10$ and $\geq 15$-letter gain in BCVA, respectively. ${ }^{85}$

\section{RPE transplantation}

Two separate phase I/II trials have evaluated safety of RPE transplantation in patients with advanced dry AMD-related GA (NCT01344993) and Stargardt disease (NCT01345006). In the AMD trial $(n=9)$, RPE cells derived from human embryonic stem cells (MA09-hRPE) were delivered to the subretinal space after standard vitrectomy. There was no evidence of adverse proliferation, rejection or serious ocular or systemic safety concerns, with the adverse events deemed to be associated to the surgery and immunosuppression. Six (66\%) out of nine patients showed a BCVA improvement of at least 11 ETDRS letters and the other three subjects remained stable (defined as change of $\leq 10$ letters) ${ }^{86}$ Combined results of the two trials showed that 
out of the 18 patients enroled with either Stargardt disease or AMD-related GA, 72\% (13/18) had patches of increased subretinal pigmentation at the border of the atrophic areas.

Another phase I/IIa trial ( $\mathrm{n}=16$; NCT02590692), administered a polarised monolayer of human embryonic stem cellderived RPE cells (CPCB-RPE1) on a parylene membrane in the subretinal space of subjects with GA. Interim data revealed that the product was successfully implanted in four subjects, with an improvement of 17 ETDRS letters in one patient. None of the implanted eyes showed progression of vision loss after 1 year of follow-up. ${ }^{87}$

\section{Mitochondrial enhancers}

Elamipretide is a cardiolipin-protective compound that protects the structure of the mitochondrial cristae and promotes oxidative phosphorylation. ${ }^{88}$ In a mouse model of sub-RPE deposits (ApoE4 transgenic mice fed high fat and cholesterol), mitochondrial dysfunction was suggested to be a trigger of deposit formation, which was successfully reversed with subcutaneous elamipretide. ${ }^{89}$ The ReCLAIM study was a phase I trial $(n=40$; NCT02848313), designed to evaluate the safety and tolerability of subcutaneous elamipretide in patients with intermediate AMD (including a high-risk drusen without GA subgroup and a noncentral GA subgroup). The outcomes were assessed at week 24. Patients with non-central GA $(\mathrm{n}=15)$ showed a mean increase in low-luminance visual acuity of $5.4 \pm 7.9$ letters and BCVA of $4.6 \pm 5.1$ letters. The patients with high-risk drusen $(n=19)$ also demonstrated improvements in low luminance and BCVA. Adverse events were mostly limited to reactions in the injection site. ${ }^{90}$ A placebo-controlled phase II trial, ReCLAIM-2 ( $\mathrm{n}=180$; NCT03891875) is currently enrolling.

\section{Nanosecond laser therapy}

The capacity of subthreshold nanosecond laser treatment to reduce drusen and reduce Bruch's membrane thickness, while maintaining retinal structure, was assessed in the Laser intervention in early stages of AMD (LEAD; $n=292$; NCT01790802) randomised trial, after promising results in preclinical studies. ${ }^{91}$ Overall, there was no significant difference in the progression rate to late AMD between those receiving nanosecond laser and the sham group. A post hoc analysis showed evidence of effect modification based on the coexistence of reticular pseudodrusen (RPD), where progression was slowed down after the laser treatment in participants without coexistent RPD at baseline and accelerated in those with RPD. ${ }^{92}$

\section{CONCLUSIONS}

AMD is a complex disease with several pathways plausibly involved in its pathogenesis, which poses therapeutic challenges. For the time being, the management of dry AMD depends greatly on observation, lifestyle changes, frequent follow-up evaluations, early recognition of visual deterioration and $\mathrm{CNV}$ detection. Several therapeutic avenues to reduce the rate of disease progression are being investigated, including (1) drugs with antioxidative properties, (2) inhibitors of the complement cascade, (3) neuroprotective agents, (4) visual cycle inhibitors, (5) gene therapy and (6) cell-based therapies, among others.

Several early phase clinical trials have shown significant promise, creating high expectations from the associated expansion phases that are ongoing or anticipated in the near future. In contrast, several trials have failed to meet primary and secondary endpoints, despite promising results in animal models and ex vivo. The main difficulties have revolved around the various methods of drug delivery, the lack of efficacy in reducing the rate of atrophy progression as compared with control eyes, and safety challenges. It is still unclear if some of the investigational agents used to treat dry AMD can predispose or accelerate the rate of progression to nAMD.

Nevertheless, given the enormous and increasing burden of disease that dry AMD represents, continued rapid development is expected, with the number of clinical trials increasing into the foreseeable future-and optimism that a meaningful treatment will be approved in the near future.

Funding This work has been supported by grants from the National Institute for Health Research Biomedical Research Centre at Moorfields Eye Hospital NHS Foundation Trust and UCL Institute of Ophthalmology, Moorfields Eye Charity, Retina UK, the Foundation Fighting Blindness (USA) and The Wellcome Trust [099173/Z/12/Z]. For the purpose of open access, the author has applied a CC BY public copyright licence to any Author Accepted Manuscript version arising from this submission.

Disclaimer The views expressed in the submitted article are our own and not an official position of the institution.

Competing interests The authors alone are responsible for the content and writing of this article. MM and MG consult for MeiraGTx Ltd.

Patient consent for publication Not required.

Provenance and peer review Not commissioned; externally peer reviewed.

Open access This is an open access article distributed in accordance with the Creative Commons Attribution 4.0 Unported (CC BY 4.0) license, which permits others to copy, redistribute, remix, transform and build upon this work for any purpose, provided the original work is properly cited, a link to the licence is given, and indication of whether changes were made. See: https://creativecommons.org/ licenses/by/4.0/.

\section{ORCID iDs}

Thales Antonio Cabral de Guimaraes http://orcid.org/0000-0002-7936-6851

Malena Daich Varela http://orcid.org/0000-0003-4960-4510

Michalis Georgiou http://orcid.org/0000-0001-6397-8071

\section{REFERENCES}

1 Flaxman SR, Bourne RRA, Resnikoff S, et al. Global causes of blindness and distance vision impairment 1990-2020: a systematic review and meta-analysis. Lancet Glob Health 2017;5:e1221-34.

2 Wong WL, Su X, Li X, et al. Global prevalence of age-related macular degeneration and disease burden projection for 2020 and 2040: a systematic review and metaanalysis. Lancet Glob Health 2014;2:e106-16.

3 Cruess AF, Zlateva $G$, Xu X, et al. Economic burden of bilateral neovascular agerelated macular degeneration: multi-country observational study. Pharmacoeconomics 2008;26:57-73.

4 Kim S, Park SJ, Byun SJ, et al. Incremental economic burden associated with exudative age-related macular degeneration: a population-based study. BMC Health Serv Res 2019;19:828.

5 Guimaraes TACde, Georgiou M, Bainbridge JWB, et al. Gene therapy for neovascular age-related macular degeneration: rationale, clinical trials and future directions. $\mathrm{Br} J$ Ophthalmol 2021;105:151-7.

6 Klein R, Davis MD, Magli YL, et al. The Wisconsin age-related maculopathy grading system. Ophthalmology 1991;98:1128-34.

7 Bird AC, Bressler NM, Bressler SB, et al. An international classification and grading system for age-related maculopathy and age-related macular degeneration. The International arm epidemiological study group. Surv Ophthalmol 1995;39:367-74.

8 Sadda SR, Guymer R, Holz FG, et al. Consensus definition for atrophy associated with age-related macular degeneration on OCT: classification of atrophy report 3. Ophthalmology 2018;125:537-48.

9 Ferris FL, Wilkinson CP, Bird A, et al. Clinical classification of age-related macular degeneration. Ophthalmology 2013;120:844-51.

10 DeAngelis MM, Owen LA, Morrison MA, et al. Genetics of age-related macular degeneration (AMD). Hum Mol Genet 2017;26:R45-50.

11 Datta S, Cano M, Ebrahimi K, et al. The impact of oxidative stress and inflammation on RPE degeneration in non-neovascular AMD. Prog Retin Eye Res 2017;60:201-18.

12 Cho E, Hung S, Willett WC, et al. Prospective study of dietary fat and the risk of agerelated macular degeneration. Am J Clin Nutr 2001;73:209-18.

13 Klein R, Klein BE, Moss SE. Relation of smoking to the incidence of age-related maculopathy. The Beaver dam eye study. Am J Epidemiol 1998;147:103-10.

14 Smith W, Mitchell P, Leeder SR. Smoking and age-related maculopathy. the blue Mountains eye study. Arch Ophthalmol 1996;114:1518-23. 
15 Anderson DH, Mullins RF, Hageman GS, et al. A role for local inflammation in the formation of drusen in the aging eye. Am J Ophthalmol 2002;134:411-31.

16 Feigl B. Age-related maculopathy - linking aetiology and pathophysiological changes to the ischaemia hypothesis. Prog Retin Eye Res 2009;28:63-86.

17 Ebrahimi KB, Handa JT. Lipids, lipoproteins, and age-related macular degeneration. J Lipids 2011;2011:1-14

18 van Lookeren Campagne M, LeCouter J, Yaspan BL, et al. Mechanisms of age-related macular degeneration and therapeutic opportunities. J Pathol 2014;232:151-64.

19 Gehrs KM, Jackson JR, Brown EN, et al. Complement, age-related macular degeneration and a vision of the future. Arch Ophthalmol 2010;128:349-58.

20 Chirco KR, Tucker BA, Stone EM, et al. Selective accumulation of the complement membrane attack complex in aging choriocapillaris. Exp Eye Res 2016;146:393-7.

21 Zeng S, Whitmore SS, Sohn EH, et al. Molecular response of chorioretinal endothelial cells to complement injury: implications for macular degeneration. J Pathol 2016:238:446-56.

22 Whitmore SS, Sohn EH, Chirco KR, et al. Complement activation and choriocapillaris loss in early AMD: implications for pathophysiology and therapy. Prog Retin Eye Res 2015;45:1-29.

23 Mullins RF, Johnson MN, Faidley EA, et al. Choriocapillaris vascular dropout related to density of drusen in human eyes with early age-related macular degeneration. Invest Ophthalmol Vis Sci 2011;52:1606-12.

24 Mullins RF, Dewald AD, Streb LM, et al. Elevated membrane attack complex in human choroid with high risk complement factor $\mathrm{H}$ genotypes. Exp Eye Res 2011;93:565-7.

25 Ding X, Patel M, Chan C-C. Molecular pathology of age-related macular degeneration. Prog Retin Eye Res 2009;28:1-18.

26 Fritsche LG, Chen W, Schu M, et al. Seven new loci associated with age-related macular degeneration. Nat Genet 2013;45:433-9.

27 Sangiovanni JP, Agrón E, Meleth AD, et al. \{omega\}-3 Long-chain polyunsaturated fatty acid intake and 12-y incidence of neovascular age-related macular degeneration and central geographic atrophy: AREDS report 30, a prospective cohort study from the Age-Related Eye Disease Study. Am J Clin Nutr 2009:90:1601-7.

28 Age-Related Eye Disease Study 2 Research Group. Lutein + zeaxanthin and omega-3 fatty acids for age-related macular degeneration: the age-related eye disease study 2 (AREDS2) randomized clinical trial. JAMA 2013:309:2005-15.

29 Age-Related Eye Disease Study 2 (AREDS2) Research Group, Chew EY, Clemons TE, et al. Secondary analyses of the effects of lutein/zeaxanthin on age-related macular degeneration progression: AREDS2 report No. 3. JAMA Ophthalmol 2014;132:142-9.

30 Evans JR, Lawrenson JG. Antioxidant vitamin and mineral supplements for slowing the progression of age-related macular degeneration. Cochrane Database Syst Rev 2017:7:CD000254.

31 Tanito M, Li F, Anderson RE. Protection of retinal pigment epithelium by OT-551 and its metabolite TEMPOL-H against light-induced damage in rats. Exp Eye Res 2010:91:111-4

32 Wong WT, Kam W, Cunningham D, et al. Treatment of geographic atrophy by the topical administration of OT-551: results of a phase II clinical trial. Invest Ophthalmol Vis Sci 2010;51:6131-9.

33 Rammes G, Gravius A, Ruitenberg M, et al. MRZ-99030 - A novel modulator of $A \beta$ aggregation: II - Reversal of $A \beta$ oligomer-induced deficits in long-term potentiation (LTP) and cognitive performance in rats and mice. Neuropharmacology 2015;92:170-82.

34 Rosenfeld PJ, Berger B, Reichel E, et al. A randomized phase 2 study of an antiamyloid $\beta$ monoclonal antibody in geographic atrophy secondary to age-related macular degeneration. Ophthalmol Retina 2018:2:1028-40.

35 Efficacy Cgov. Safety and tolerability study of RN6G in subjects with geographic atrophy secondary to age-related macular degeneration, 2016. Available: https:// clinicaltrials.gov/ct2/show/NCT01577381 [Accessed 20 Aug 2020].

36 Parsons CG, Ruitenberg M, Freitag CE, et al. MRZ-99030 - A novel modulator of A $\beta$ aggregation: I - Mechanism of action (MoA) underlying the potential neuroprotective treatment of Alzheimer's disease, glaucoma and age-related macular degeneration (AMD). Neuropharmacology 2015;92:158-69.

37 Galimedix Therapeutics. Phase I completed, 2020. Available: https://www.galimedix. com/clinical-trials [Accessed 20 Aug 2020].

38 GlobeNewswire. Galimedix therapeutics presents data showing safety, tolerability and potential for efficacy of its investigational eyedrops containing GAL-101 at glaucoma 360 conference, 2019. Available: https://www.globenewswire.com/ news-release/2019/02/06/1711498/0/en/Galimedix-Therapeutics-Presents-DataShowing-Safety-Tolerability-and-Potential-for-Efficacy-of-Its-InvestigationalEyedrops-Containing-GAL-101-at-Glaucoma-360-Conference.html [Accessed 20 Aug 2020].

39 Rosenfeld PJ, Dugel PU, Holz FG, et al. Emixustat hydrochloride for geographic atrophy secondary to age-related macular degeneration: a randomized clinical trial. Ophthalmology 2018;125:1556-67.

40 Radu RA, Han Y, Bui TV, et al. Reductions in serum vitamin A arrest accumulation of toxic retinal fluorophores: a potential therapy for treatment of lipofuscin-based retinal diseases. Invest Ophthalmol Vis Sci 2005;46:4393-401.

41 Mata NL, Lichter JB, Vogel R, et al. Investigation of oral fenretinide for treatment of geographic atrophy in age-related macular degeneration. Retina 2013;33:498-507.
$42 \mathrm{Ma} \mathrm{L}$, Kaufman Y, Zhang J, et al. C20-D3-vitamin a slows lipofuscin accumulation and electrophysiological retinal degeneration in a mouse model of Stargardt disease. J Biol Chem 2011;286:7966-74

43 Kaufman Y, Ma L, Washington I. Deuterium enrichment of vitamin A at the C20 position slows the formation of detrimental vitamin A dimers in wild-type rodents. J Biol Chem 2011:286:7958-65.

44 Hillmen P, Young NS, Schubert J, et al. The complement inhibitor eculizumab in paroxysmal nocturnal hemoglobinuria. N Eng/ J Med 2006;355:1233-43.

45 Maga TK, Nishimura CJ, Weaver AE, et al. Mutations in alternative pathway complement proteins in American patients with atypical hemolytic uremic syndrome. Hum Mutat 2010;31:E1445-60.

46 Yehoshua Z, de Amorim Garcia Filho CA, Nunes RP, et al. Systemic complement inhibition with eculizumab for geographic atrophy in age-related macular degeneration: the complete study. Ophthalmology 2014;121:693-701.

47 Loyet KM, Good J, Davancaze T, et al. Complement inhibition in cynomolgus monkeys by anti-factor $D$ antigen-binding fragment for the treatment of an advanced form of dry age-related macular degeneration. J Pharmacol Exp Ther 2014;351:527-37

48 Katschke KJ, Wu P, Ganesan R, et al. Inhibiting alternative pathway complement activation by targeting the factor D exosite. J Biol Chem 2012;287:12886-92.

49 Holz FG, Sadda SR, Busbee B, et al. Efficacy and safety of Lampalizumab for geographic atrophy due to age-related macular degeneration: Chroma and Spectri phase 3 randomized clinical trials. JAMA Ophthalmol 2018;136:61666-77.

50 Camardo J. The rapamune era of immunosuppression 2003: the journey from the laboratory to clinical transplantation. Transplant Proc 2003;35:S18-24.

51 Wong WT, Dresner S, Forooghian F, et al. Treatment of geographic atrophy with subconjunctival sirolimus: results of a phase I/II clinical trial. Invest Ophthalmol Vis Sci 2013:54:2941-50.

52 Jaffe GJ, Westby K, Csaky KG. C5 inhibitor Avacincaptad pegol for geographic atrophy due to age-related macular degeneration: a randomized pivotal phase 2/3 trial. Ophthalmology 2020;S0161-6420:30845-9.

53 Csaky KG, Westby K, Rezaei K. Avacincaptad pegol, a novel C5 inhibitor, significantly reduces the mean rate of geographic atrophy growth in a pivotal clinical trial. Invest Ophthalmol Vis Sci 2020;61:1943.

54 IVERIC bio. IVERIC BIO Announces positive Zimura 18 month data supporting the 12 month efficacy findings: continuous positive treatment effect with favorable safety profile in geographic atrophy secondary to age-related macular degeneration in a phase 3 trial, 2020. Available: https://www.prnewswire.com/ news-releases/iveric-bio-announces-positive-zimura-18-month-data-supportingthe-12-month-efficacy-findings-continuous-positive-treatment-effect-withfavorable-safety-profile-in-geographic-atrophy-secondary-to-age-related-macular [Accessed 2 Aug 2020].

55 Liao DS, Grossi FV, El Mehdi D, et al. Complement C3 inhibitor Pegcetacoplan for geographic atrophy secondary to age-related macular degeneration: a randomized phase 2 trial. Ophthalmology 2020;127:186-95.

56 News, Healio. Apellis completes phase 3 study enrollment of geographic atrophy treatment, 2020. Available: https://www.healio.com/news/ophthalmology/20200708/ apellis-completes-phase-3-study-enrollment-of-geographic-atrophy-treatment [Accessed 30 Jul 2020].

57 News, Healio. Anti-complement C5 monotherapy ineffective in reducing geographic atrophy lesion size, 15 Feb 2016. Available: https://www.healio.com/news/ ophthalmology/20160215/anticomplement-c5-monotherapy-ineffective-in-reducinggeographic-atrophy-lesion-size [Accessed 30 Jul 2020].

58 Shaw LT, Mackin A, Shah R, et al. Risuteganib-a novel integrin inhibitor for the treatment of non-exudative (dry) age-related macular degeneration and diabetic macular edema. Expert Opin Investig Drugs 2020;29:547-54.

59 Charters L. Risuteganib: Exporing novel dry AMD treatment. ophthalmology times, 2020. Available: https://www.ophthalmologytimes.com/view/risuteganib-exploringnovel-dry-amd-treatment

60 Oveson BC, Iwase T, Hackett SF, et al. Constituents of bile, bilirubin and TUDCA, protect against oxidative stress-induced retinal degeneration. J Neurochem 2011;116:144-53.

61 Lawson EC, Bhatia SK, Han MK, et al. Tauroursodeoxycholic acid protects retinal function and structure in RD1 mice. Adv Exp Med Biol 2016:854:431-6.

62 Phillips MJ, Walker TA, Choi H-Y, et al. Tauroursodeoxycholic acid preservation of photoreceptor structure and function in the rd10 mouse through postnatal day 30 . Invest Ophthalmol Vis Sci 2008;49:2148-55

63 Shibagaki K, Okamoto K, Katsuta O, et al. Beneficial protective effect of pramipexole on light-induced retinal damage in mice. Exp Eye Res 2015;139:64-72.

64 Wen R, Tao W, Li Y, et al. Cntf and retina. Prog Retin Eye Res 2012;31:136-51.

65 Pardue MT, Allen RS. Neuroprotective strategies for retinal disease. Prog Retin Eye Res 2018:65:50-76

66 Sieving PA, Caruso RC, Tao W, et al. Ciliary neurotrophic factor (CNTF) for human retinal degeneration: phase I trial of CNTF delivered by encapsulated cell intraocula implants. Proc Natl Acad Sci U S A 2006;103:3896-901.

67 Kauper K, McGovern C, Sherman S, et al. Two-year intraocular delivery of ciliary neurotrophic factor by encapsulated cell technology implants in patients with chronic retinal degenerative diseases. Invest Ophthalmol Vis Sci 2012;53:7484-91. 
68 Krupin T, Liebmann JM, Greenfield DS, et al. A randomized trial of brimonidine versus timolol in preserving visual function: results from the low-pressure glaucoma treatment study. Am J Ophthalmol 2011;151:671-81.

69 Merin S, Obolensky A, Farber MD, et al. A pilot study of topical treatment with an alpha2-agonist in patients with retinal dystrophies. J Ocul Pharmacol Ther 2008;24:80-6.

70 Lafuente MP, Villegas-Pérez MP, Sobrado-Calvo P, et al. Neuroprotective effects of alpha(2)-selective adrenergic agonists against ischemia-induced retinal ganglion cell death. Invest Ophthalmol Vis Sci 2001;42:2074-84.

71 Donello JE, Padillo EU, Webster ML, et al. alpha(2)-adrenoceptor agonists inhibit vitreal glutamate and aspartate accumulation and preserve retinal function after transient ischemia. J Pharmacol Exp Ther 2001;296:216-23.

72 Yoles E, Wheeler LA, Schwartz M. Alpha2-adrenoreceptor agonists are neuroprotective in a rat model of optic nerve degeneration. Invest Ophthalmol Vis Sci 1999;40:65-73.

73 WoldeMussie E, Ruiz G, Wijono M, et al. Neuroprotection of retinal ganglion cells by brimonidine in rats with laser-induced chronic ocular hypertension. Invest Ophthalmol Vis Sci 2001;42:2849-55.

74 Rajagopalan L, Ghosn C, Tamhane M. Cyto-/neuro-protective effects of brimonidine drug delivery system (DDS) in a nonhuman primate progressive retinal degeneration model of geographic atrophy $(\mathrm{GA})$ secondary to age-related macular degeneration (AMD). Invest Ophthalmol Vis Sci 2019;60:2993.

75 Kuppermann BD, Patel SS, Boyer DS, et al. Phase 2 study of the safety and efficacy of brimonidine drug delivery system (Brimo DDS) generation 1 in patients with geographic atrophy secondary to age-related macular degeneration. Retina 2021:41:144-55.

76 Qian CX. AAO retina Subday: first-time phase II results of Brimo-DDS for geographic atrophy, 2018. Available: https://retinaroundup.com/2018/10/28/2018-aao-retinasubday-first-time-phase-ii-results-of-brimo-dds-for-geographic-atrophy/ [Accessed 17 Aug 2020].

77 Freeman WR. Brimonidine drug delivery system for geographic atrophy. An implant with potential to slow lesion growth, 2019. Available: https://www.retinalphysician com/issues/2019/november-2019/brimonidine-drug-delivery-system-for-geographicat\#reference-8 [Accessed 17 Aug 2020].

78 Kumar-Singh R. The role of complement membrane attack complex in dry and wet AMD - From hypothesis to clinical trials. Exp Eye Res 2019;184:266-77.

79 Cashman SM, Ramo K, Kumar-Singh R. A non membrane-targeted human soluble CD59 attenuates choroidal neovascularization in a model of age related macular degeneration. PLoS One 2011;6:e19078.
80 Dugel PU. CLINICAL TRIAL DOWNLOAD: Data on a Gene Therapy for Dry and Wet AMD, 2020. Available: https://www.retinalphysician.com/issues/2020/april-2020/ clinical-trial-download-data-on-a-gene-therapy-for [Accessed 02 Aug 2020].

81 Lachmann PJ. The story of complement factor I. Immunobiology 2019;224:511-7.

82 Ellis S, Buchberger A, Holder J. GT005, a gene therapy for the treatment of dry agerelated macular degeneration (AMD). Invest Ophthalmol Vis Sci 2020;61.

83 Cashman SM, Gracias J, Adhi M, et al. Adenovirus-mediated delivery of factor $\mathrm{H}$ attenuates complement $\mathrm{C} 3$ induced pathology in the murine retina: a potential gene therapy for age-related macular degeneration. J Gene Med 2015;17:229-43.

84 Lund RD, Wang S, Lu B, et al. Cells isolated from umbilical cord tissue rescue photoreceptors and visual functions in a rodent model of retinal disease. Stem Cells 2007;25:1089-11.

85 Ho AC, Chang TS, Samuel M, et al. Experience with a subretinal cell-based therapy in patients with geographic atrophy secondary to age-related macular degeneration. Am J Ophthalmol 2017;179:67-80.

86 Schwartz SD, Regillo CD, Lam BL, et al. Human embryonic stem cell-derived retinal pigment epithelium in patients with age-related macular degeneration and Stargardt's macular dystrophy: follow-up of two open-label phase 1/2 studies. Lancet 2015;385:9967:509-16.

87 Kashani AH, Lebkowski JS, Rahhal FM, et al. A bioengineered retinal pigment epithelial monolayer for advanced, dry age-related macular degeneration. Sci Trans/ Med 2018; 10:eaa04097.

88 Szeto HH. First-in-class cardiolipin-protective compound as a therapeutic agent to restore mitochondrial bioenergetics. Br J Pharmacol 2014;171:2029-50.

89 Cousin SW, Saloupis P, Brahmajoti MV. Mitochondrial dysfunction in experimental mouse models of SubRPE deposit formation and reversal by the Mito-Reparative drug MTP-131. Invest Ophthalmol Vis Scie 2016;57.

90 Stealth BioTherapeutics. Stealth biotherapeutics announces positive results for elamipretide in ophthalmic conditions, 2019. Available: https://www.prnewswire.com/ news-releases/stealth-biotherapeutics-announces-positive-results-for-elamipretide-inophthalmic-conditions-300841246.html [Accessed 20 Aug 2020].

91 Jobling Al, Guymer RH, Vessey KA, et al. Nanosecond laser therapy reverses pathologic and molecular changes in age-related macular degeneration without retinal damage. Faseb J 2015;29:696-710.

92 Guymer RH, Wu Z, Hodgson LAB, et al. Subthreshold nanosecond laser intervention in age-related macular degeneration: the lead randomized controlled clinical trial. Ophthalmology 2019;126:829-38. 